\title{
Theranostics of Neuroendocrine Tumors
}

\author{
Sze Ting Lee ${ }^{a} \quad$ Harshad R. Kulkarni $^{b} \quad$ Aviral Singh $^{\mathrm{b}} \quad$ Richard P. Baum $^{\mathrm{b}}$ \\ a Department of Molecular Imaging and Therapy, Austin Health, Heidelberg, Melbourne, VIC, Australia; \\ bTHERANOSTICS Center for Molecular Radiotherapy and Molecular Imaging, ENETS Center of Excellence, Zentralklinik Bad Berka, \\ Bad Berka, Germany
}

\section{Keywords}

Peptide receptor radionuclide therapy, PRRT .

Neuroendocrine tumor, NET - Theranostics - Diagnosis . Positron emission tomography/computed tomography, $\mathrm{PET} / \mathrm{CT}$

\section{Summary}

Somatostatin receptor positron emission tomography/ computed tomography using ${ }^{68} \mathrm{Ga}$-labeled somatostatin analogs is the mainstay for the evaluation of receptor status in neuroendocrine tumors (NETs). This translates towards better therapy options, with increasing evidence of peptide receptor radionuclide therapy (PRRT) as the treatment of choice for advanced or progressive NETs. There are benefits in progression-free and overall survival as well as a significant improvement in clinical condition. In patients with progressive NETs, fractionated, personalized PRRT results in good therapeutic responses with no significant severe hematological and/or renal toxicity, thus improving quality of life.

(C) 2017 S. Karger GmbH, Freiburg

\section{Introduction}

The term 'theranostics' is derived from the combination of two words, i.e. therapy and diagnostics, which are integral in the current era of personalized medicine. In nuclear medicine, this has been utilized in the treatment of thyroid carcinoma with radioiodine ablation for decades, where the radioiodine diagnostic scan is used to determine the treatment dose of radioiodine. In the more contemporary practice of nuclear medicine, the use of molecular targets (e.g. antibodies or peptides) labeled with diagnostic or therapeutic radionuclides can be used for the diagnosis and therapy of a particular disease at a molecular level.
Neuroendocrine neoplasm or neuroendocrine tumor (NET) is a heterogeneous group of epithelial neoplasms predominantly characterized by neuroendocrine differentiation $[1,2]$. These tumors are characterized by high expression of somatostatin receptors (SSTRs) and specific markers, including chromogranin A, CD53 protein, and neuron-specific enolase [3]. Currently, NET is classified based on the grading (Ki67 and mitotic index (MI)) and location of the neoplasms. Although NETs can originate from any region of the body, $60-70 \%$ derive from the gastroenteropancreatic system (GEP-NET). Approximately 30\% of NETs have associated symptoms; therefore, they are considered as functioning tumors. These symptoms are often related to the hormonal hypersecretion, which frequently include diarrhea, flushing, hyperglycemia, or hypoglycemia. NETs of the lungs are also known as pulmonary carcinoid tumors, which account for $1-2 \%$ of all lung tumors and approximately $30 \%$ of all NETs. Pulmonary NETs are classified according to their mitotic count rate and the presence of necrosis [2].

The new NET classification guidelines for GEP-NET (4th edition) of the World Health Organization (WHO) from 2017 are based on the proliferation (Ki67 and MI) and differentiation (table 1). Well-differentiated tumors are grade 1 (Ki67 <3\% and MI $<2 / 10$ high power field (HPF)), grade 2 (Ki67 3-20\% and MI $2-20 / 10 \mathrm{HPF}$ ), and grade 3 (Ki67 $>20 \%$ and $\mathrm{MI}>20 / 10 \mathrm{HPF}$ ). There is a subdivision of tumors with a $\mathrm{Ki} 67>20 \%$ and an MI $>20 / 10$ HPF into well-differentiated grade 3 NET and poorly differentiated neuroendocrine carcinoma (NEC), the latter of which are divided into small cell and large cell carcinomas. There is also a new category of mixed neuroendocrine and non-neuroendocrine neoplasm (MiNET). The rationale for the differentiation of grade 3 NETs is that some grade 3 tumors which are classified into this category according to the Ki67 index have been recognized to behave more like grade 2 NETs rather than aggressive carcinomas. The NORDIC NEC study showed that although patients with a Ki67 $<55 \%$ were less responsive to platinum-based chemotherapy, they had a longer survival, and therefore not all NEC should be considered as one single disease entity.

\section{KARGER}

(๑) 2017 S. Karger GmbH, Freiburg

Fax +497614520714
Prof. Dr. Richard P. Baum

THERANOSTICS Center for Molecular Radiotherapy and Molecular Imaging (PET/CT) ENETS Center of Excellence, Zentralklinik Bad Berka

Robert-Koch-Allee 9, 99437 Bad Berka, Germany

richard.baum @ zentralklinik.de 
Theranostics of NETs includes diagnosis of the disease with positron emission tomography/computed tomography (PET/CT) using Ga-68-labeled somatostatin analogs, which bind specifically to different SSTR subtypes and allow the molecular imaging and characterization of NETs, with a very high diagnostic sensitivity and specificity for the early identification of metastases $[4,5]$. This has also been shown to have a high impact on patient management [6]. In pulmonary NETs, SSTR PET/CT has been shown to have a significant impact on treatment strategy in up to $18 \%$ of patients [7]. This subsequently allows the best management approach for each patient [8] as well as the evaluation of treatment response post-therapy [9]. Following the determination of SSTR expression using PET/CT, peptide receptor radionuclide therapy (PRRT) can be instituted using therapeutic radionuclides (e.g. beta- or alphaemitting radioisotopes) labeled with the same tracer for personalized treatment. This is accompanied by the ability to assess the patient dosimetry with pre- as well as post-therapy imaging and the assessment of therapy response using imaging by means of further Ga-68 SSTR PET/CT.

\section{Different Radionuclides for Peptide Receptor Radionuclide Therapy}

Initially, PRRT in NETs was performed with ${ }^{111}$ In-pentetreotide, which had a short tissue penetration of the emitted Auger electrons (ranging between nanometers and micrometers). Initial study of 50 patients had shown that there was a clinical benefit and safety with regards to renal function; however, leukemia or myelodysplastic syndrome (MDS) was reported in 3 patients who received $>100 \mathrm{GBq}$ of ${ }^{111}$ In-pentetreotide [10]. This treatment is still used at some centers in the USA mainly due to the lack of accessibility to beta-emitting radiotracers.

Subsequently, beta-emitters such as Y-90 (maximum energy (Emax) 2.27 mega-electronvolts $(\mathrm{MeV})$, half-life $64 \mathrm{~h}$, tissue penetration 2.5-11 mm) and Lu-177 (Emax 0.49 MeV, half-life 6.7 days, tissue penetration $2 \mathrm{~mm}$ ) were used to link to octreotide. The initial studies on Y-90-DOTA-Tyr3-octreotide (Y-90 DOTATOC) had reported objective response rates in $6-37 \%$ of patients, and response to Y-90 DOTATOC was associated with a longer survival. SSTR imaging was predictive for both survival after Y-90 DOTATOC therapy and the occurrence of renal toxicity $[3,11]$. Whilst Lu-177-DOTA-Tyr3-octreotate (Lu-177 DOTATATE) utilized the lower tissue penetration of Lu-177 coupled with the longer half-life of the radionuclide, DOTATATE has up to 9-fold higher peptide receptor affinity for SSTR2 compared to DOTATOC [12], the latter having higher affinity to SSTR 3 and SSTR5 [13]. The high gamma-emission from Lu-177 allows for high-quality post-treatment images which would be an accurate representation of the distribution of the radionuclide therapy in all the lesions, and can be used to monitor the uptake in between treatments. Clinical trials using Lu-177 DOTATATE have shown a high efficacy and tolerability, especially with regard to the kidneys. Although the uptake, residence time, and mean absorbed renal dose per unit of adminis-
Table 1. Classification of neuroendocrine neoplasms of the gastroenteropancreatic system (WHO 2017) ${ }^{\mathrm{a}}$

\begin{tabular}{|c|c|c|c|}
\hline Grade & Description & Ki-67, \% & Mitotic index (HPF) \\
\hline G1 & well-differentiated NEN & $<2$ & $<2 / 10$ \\
\hline G2 & well-differentiated NEN & $3-20$ & $2-20 / 10$ \\
\hline G3 & well-differentiated NEN & $>20$ & $>20 / 10$ \\
\hline $\mathrm{G}^{\mathrm{b}}$ & $\begin{array}{l}\text { poorly differentiated NEN } \\
\text { or NEC }\end{array}$ & $>20$ & $\begin{array}{l}\text { bdifferentiated into } \\
\text { small and large cell }\end{array}$ \\
\hline
\end{tabular}

${ }^{a}$ New category: mixed NEN/non-NEN (MiNEN), hyperplastic and preneoplastic lesions.

NEN = Neuroendocrine neoplasm (also called NET = neuroendocrine tumor); $\mathrm{NEC}=$ neuroendocrine carcinoma; $\mathrm{HPF}=$ high power field.

tered activity is higher for DOTATATE, both peptides were found to be safe in terms of potential for renal toxicity [14]. Lu-177 DOTATATE might have more favorable characteristics for PRRT due to a lower whole-body dose, resulting in potentially lower bone marrow toxicity [15]. Dosimetry studies have also confirmed the high specificity of PRRT against tumors, whilst being able to spare healthy tissue [3]. Current administration schedules comprise the most appropriate number of cycles (generally four or five) and the best delivery frequency (10 \pm 2 weeks apart). This treatment periodicity allows the patient to recover from the mild side effects of the therapy and increases its effectiveness in terms of the radiobiological activity [16].

\section{Patient Selection}

The most common first-line treatment of NETs would usually include complete surgical resection of the primary tumor \pm regional lymph node metastases, followed by cold somatostatin analogs. The last two decades have seen the establishment of PRRT as an effective treatment option for patients with inoperable or metastatic well-differentiated NETs who have progressed with cold somatostatin therapy.

The WHO grade of the tumor determined by the Ki67 tumor proliferation index is essential for patient selection [17], with G1 and G2 tumors known to respond well to PRRT. High SSTR expression of tumors must also be ascertained before PRRT to ensure therapeutic efficacy; this is assessed by using Tc-99m-labeled somatostatin analog (Tektrotyd ${ }^{\mathrm{TM}}$; ROTOP Pharmaka GmbH, Dresden, Germany) or, much better, with SSTR PET/CT using Ga-68 DOTA peptides. On the SSTR PET/CT, the standardized uptake value (SUV) of the tumors on PET/CT is another excellent, quantitative measure of the degree of SSTR expression, and could potentially assist in predicting the response to PRRT [4], thus leading to better patient selection for PRRT (table 2).

The prognostic value of ${ }^{18} \mathrm{~F}$-fluorodeoxyglucose (FDG) PET in the selection of patients for PRRT has also been assessed in $52 \mathrm{pa}-$ tients with progressive advanced well-differentiated grade 1/2 NETs treated with Lu-177 DOTATATE. It was found that none of the PET-negative patients had progressed at the first follow-up ex- 
amination after Lu-PRRT, whereas grade 2 NET and FDG PET positivity (arbitrary SUV cutoff $>2.5$ ) were frequently associated with more aggressive disease, suggesting perhaps a more intensive treatment regimen for the latter group [18]. A further study of 69 NET patients treated with Y-90 or Lu-177-DOTATOC/DOTATATE showed a partial response (PR) in $27.5 \%$ of patients, while 50.7 and $23.2 \%$ had stable disease (SD) and progressive disease (PD), respectively. Low tumor burden and a low proliferation index represent independent prognostic factors for better progression-free survival (PFS), while stage IV, NET G2, and previous transarterial chemoembolization (TACE) were found to be significant factors for tumor progression at multivariate analysis [19]. There is also evidence that FDG avidity and higher grade tumors with extensive liver involvement are important aspects to be evaluated before administering PRRT. In some situations, the efficacy of PRRT in high-risk patients can be raised by increasing the total administered activity or the number or frequency of PRRT cycles, or by administering radiosensitizer agents such as capecitabine or capecitabine plus temozolomide [20]. These new therapeutic options may provide a further personalization of treatment by increasing the dose intensity only when required [3].

There are quite a few treatment options when it comes to the treatment of NETs. These include surgical resection of the primary and localized metastases, locoregional approaches to liver metastases (including TACE and selective internal radiation therapy), chemotherapy for highly proliferating NETs, and medical treatment with SSTR agonists such as octreotide and lanreotide. The choice of the most appropriate treatment requires knowledge of the anatomical location, local disease extension, tumor functionality, SSTR status, histological grading, and staging [21]. However, PRRT is highly recommended in patients with metastatic or inoperable NETs and positive expression of SSTR2 [11, 22-25]. Although PRRT with radiolabeled somatostatin analogs is most commonly used for patients with WHO grade 1 and grade 2 gastrointestinal and bronchial NETs, patients with pheochromocytoma, paraganglioma, neuroblastoma, and medullary thyroid carcinoma may also benefit from the treatment [26-29].

\section{Types and Efficacy of Peptide Receptor Radionuclide Therapy}

PRRT in NETs has been used since 1994, where the first case report was published by the Rotterdam group, using In-111 octreotide [30]. Since then, a number of studies, mostly retrospective and monocentric, have demonstrated the efficacy of PRRT in thousands of patients.

One of the earliest retrospective studies by the Rotterdam group using Lu-177 DOTATATE in 310 GEP-NET patients showed a complete response (CR) in $3 \%$ of cases, PR in $28 \%$, and minor response (MR) in $16 \%$. The mean time to progression was 40 months and the median overall survival (OS) from diagnosis was 128 months [24]. A recent evaluation of the multi-institutional registry of patients in Germany consisting of 450 patients showed that the
Table 2. Prerequisites for peptide receptor radionuclide therapy

Histologically proven neuroendocrine tumor (immunohistochemistry) High somatostatin receptor (SSTR) expression determined by immunohistochemistry or functional imaging of SSTR (using Tc-99m-labeled somatostatin analogs, or better with positron emission tomography/computed tomography using Ga-68-labeled somatostatin analogs)

Karnofsky/Lansky performance status $>60 \%$ or ECOG $<2$

Tumor differentiation: well-differentiated, preferably G1 or G2 Proliferation rate (Ki67/mitotic index) of the tumor preferably $\leq 20 \%$

median OS of all patients was 59 months, with a median PFS of 41 months. Both OS and PFS were significantly worse in patients treated with Y-90 alone compared to Lu-177, and also in patients with grade 2 and grade 3 tumors compared to grade 1 . Complete remission was observed in $5.6 \%$ of patients, whilst $22.4 \%$ of patients had partial remissions, $47.3 \%$ of patients were stable, and $4 \%$ of patients had PD [31].

A smaller study of 74 patients showed a PR in $36.3 \%$, MR in $17.6 \%$, SD in $35.1 \%$, and PD in $10.8 \%$. The median PFS was 26 months and the median OS was 55 months. In a subgroup of 41 non-pancreatic GEP-NETs, a slightly poorer efficacy was demonstrated, with a PR of $22 \%$, MR of $17.1 \%$, SD of $48.8 \%$, and PD in $12.2 \%$ [32]. A more recent phase II study in 43 advanced, well-differentiated gastrointestinal NET (grade 1 or 2) patients treated with Lu-177 DOTATATE showed a CR in 7\% of patients and SD in $77 \%$, and a disease control rate (DCR) of $84 \%$. The median PFS was 36 months, while the median OS had not yet been reached. Once again, none of the patients showed side effects after any LuPRRT dosages [33].

Lu-177-DOTATOC has also been used with a promising efficacy and toxicity profile. A recent phase II study in 56 patients with metastatic and progressive NETs reported efficacy and safety data of Lu-177-DOTATOC. The overall median DCR was $93.8 \%$ for patients who received more than one cycle, while the median PFS and OS were 17.4 and 34.2 months, respectively, assessed over a followup time (mean \pm standard deviation) of $16.1 \pm 12.4$ months [34]. In the largest reported study to date of 1,200 patients with metastatic bronchial NETs and GEP-NETs that express SSTRs, PRRT with Lu-177-DOTATATE was demonstrated to be a favorable therapeutic option which is safe, with few side effects, and shows good response rates, with a PFS of 29 months and an OS of 63 months [35].

The most prominent results to date in a phase III study of 230 patients with progressive midgut NET treated with somatostatin analogs showed that the median PFS in those patients treated with Lu-177 DOTATATE and $30 \mathrm{mg}$ Sandostatin-LAR every 4 weeks was not reached compared to 8.4 months in patients with the highdose Sandostatin treatment alone [36]. This indicates that PRRT is very effective in this subgroup of patients (hazard ratio 0.209; $95 \%$ confidence interval 0.129-0.33).

There is also evidence that the use of both radioisotopes (Y-90 and $\mathrm{Lu}-177)$ can exploit the individual properties of the radiotracers to allow a better crossfire effect to treat this tumor group, which 
Fig. 1. 80-year-old male patient with grade I (Ki67 2\%) welldifferentiated, nonfunctioning neuroendocrine neoplasm at the rectosigmoid junction with extensive bilobar liver metastasis, diagnosed 6 years before, initial tumor stage cT3 $\mathrm{pNx}$ pM1 (HEP) stage IV, and immunohistochemical expression of chromogranin, synaptophysin, and somatostatin receptor (SSTR) IIa (in 60\%). The first symptom at diagnosis was diarrhea with sonographic suspicion of liver metastases. Liver biopsy (performed at another hospital) demonstrated poorly differentiated adenocarcinoma; however, the primary tumor could not be detected by colonoscopy. Ga-68 SSTR positron emission tomography/computed tomography (PET/CT) identified
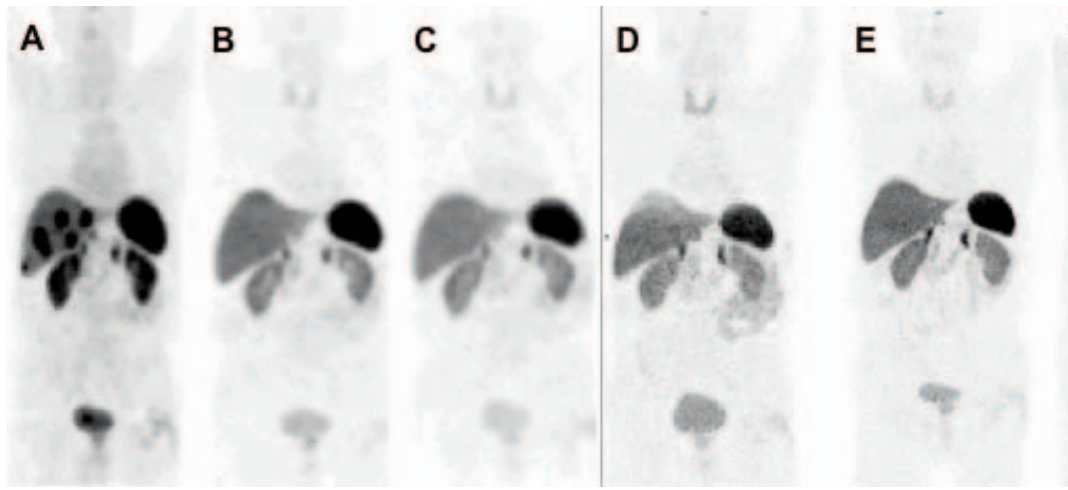

F
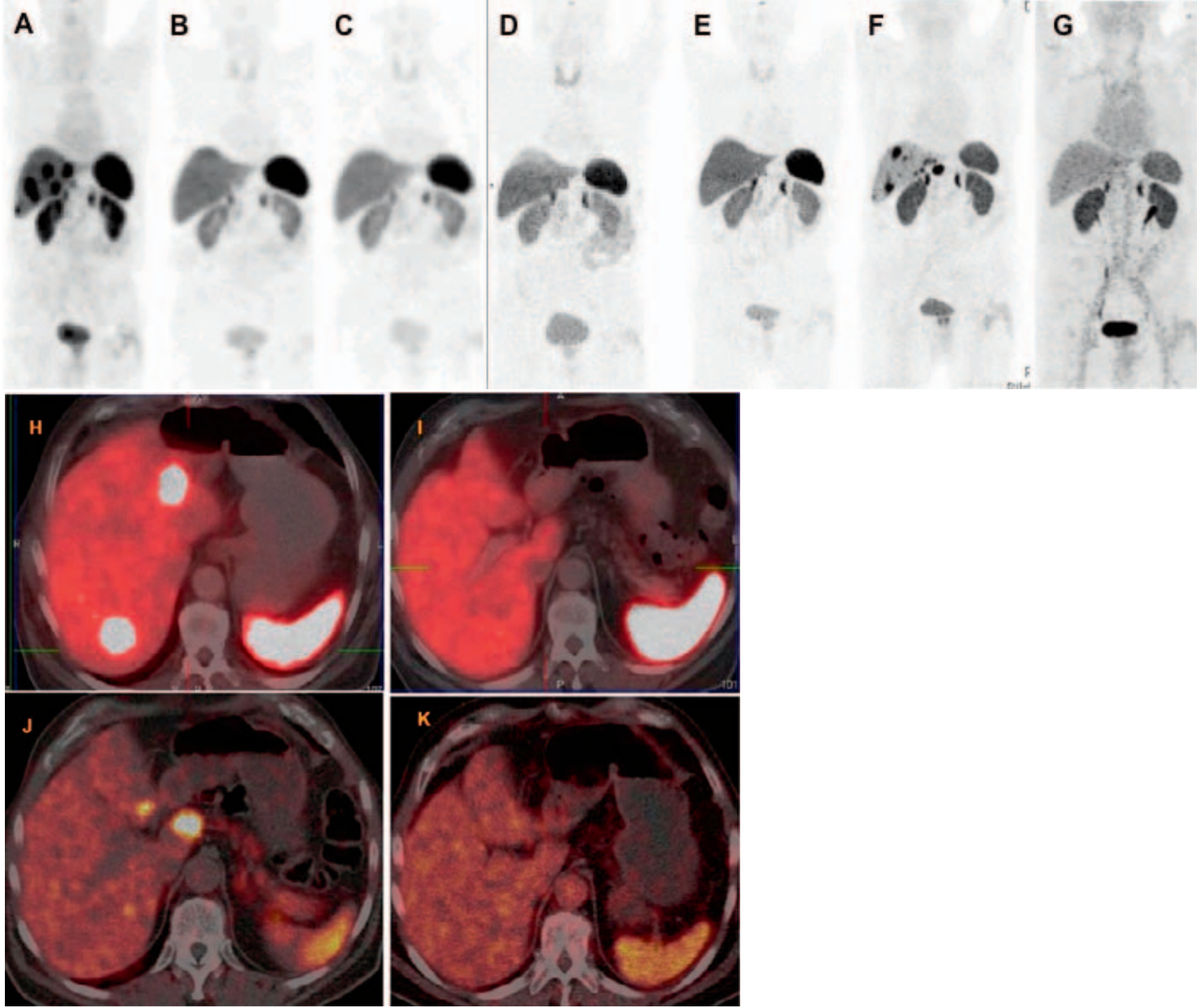

an intensely SSTR-positive tumor at the rectosigmoid junction with multiple receptor-expressing hepatic metastases (maximum size $2 \mathrm{~cm}$ ). There was no demonstrable glucose hypermetabolism in any of the tumor masses. Before referral to our center, the patient underwent systemic chemotherapy with FOLFOX and Avastin in addition to therapy with Sandostatin. There was progressive disease with continual increase in size of the liver metastases (to 3 and $3.4 \mathrm{~cm} 3$ and 9 months after initiation of chemotherapy, respectively). Three cycles of Lu-177-based peptide receptor radionuclide therapy (PRRT) were administered (also based on a review of the histopathology which revealed neuroendocrine tumors), leading to a very good response with complete remission of the liver metastases and a significant decrease in the size (on CT) and SSTR expression (PET/CT) of the primary tumor. The patient was regularly followed up, i.e. every 9-12 months, with Ga-68 SSTR PET/CT. He had a progression-free interval of 4 years after which there were new SSTR-positive liver metastases. A second phase of PRRT (consisting of the 4th and 5th cycles) was administered, resulting again in an excellent response to PRRT with complete regression of the hepatic metastases. A-G PET maximum intensity projection images of Ga-68 SSTR PET/CT; H-K fused transverse PET/CT images; A, H before PRRT; B, K 4 months after the 3rd PRRT cycle; C-E 12, 24 and 36 months, respectively, after 3rd cycle of PRRT; F, J 48 months after the 3rd PRRT cycle a new hepatic progression was demonstrated; G, K once again complete remission of the liver metastases after a second phase of PRRT (4th and 5th cycles).

demonstrates heterogeneous receptor densities. Y-90 is considered to be more suitable for larger tumors due to the higher energy and penetrating capacity of its beta particles. In this regard, Lu-177 has a lower energy and smaller particle range, resulting in better absorption in smaller tumors and therefore being less likely to be effective in large tumors, particularly given the heterogeneous distribution of SSTRs over larger tumors. The lower dose rate due to the longer half-life is also a limiting factor. Therefore, the presence of different sizes of tumors with heterogeneous distribution of SSTRs in a patient should be taken into consideration to ensure that most of the beta energy from the radioisotope is absorbed and the radiation dose to the tumor is optimized [37]. The combination of Y-90and Lu-177-labeled somatostatin analogs in an animal model has demonstrated a better tumor response than the use of each radiolabeled analog alone [38]. Therefore, DUO-PRRT, which involves sequential administration of Y-90- and Lu-177-labeled analogs, is helpful for the treatment of larger tumors, followed by subsequent treatment of smaller metastases in further treatment cycles, i.e. in two different settings 3-6 months apart. Tandem PRRT has also been performed, which specifically refers to the concurrent use of these radioisotopes in the same setting.

The results of a study of 50 patients with disseminated NETs indicated that tandem PRRT (with Y-90/Lu-177 DOTATATE) resulted in a longer OS than with a single radioisotope (Y-90 DOTATATE), and the safety of both methods were comparable [39]. Meanwhile, a more recent study of 486 patients with metastatic 
Fig. 2. Well-differentiated, functioning neuroendocrine neoplasm G2 (Ki67 10\%) of the pancreatic tail (glucagonoma) with extensive hepatic metastases (initial tumor stage IV) in a 72-yearold patient. Partial remission of disease after 4 cycles of DUO peptide receptor radionuclide therapy (PRRT) (first cycle with Y-90 and then successively using Lu-177) on Ga-68 somatostatin receptor positron emission tomography/computed tomography (PET/CT), according to both molecular and morphological imaging criteria with a significant decrease in number, size, and uptake of the multiple liver metastases as well as regression of the
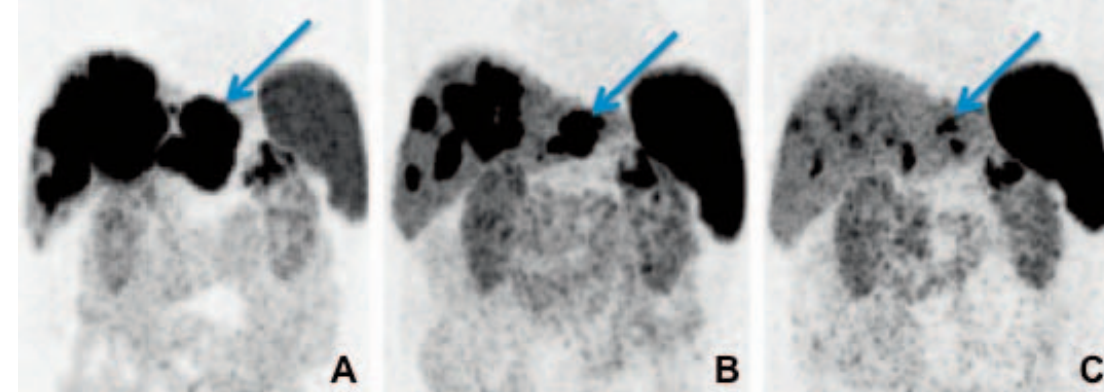

C
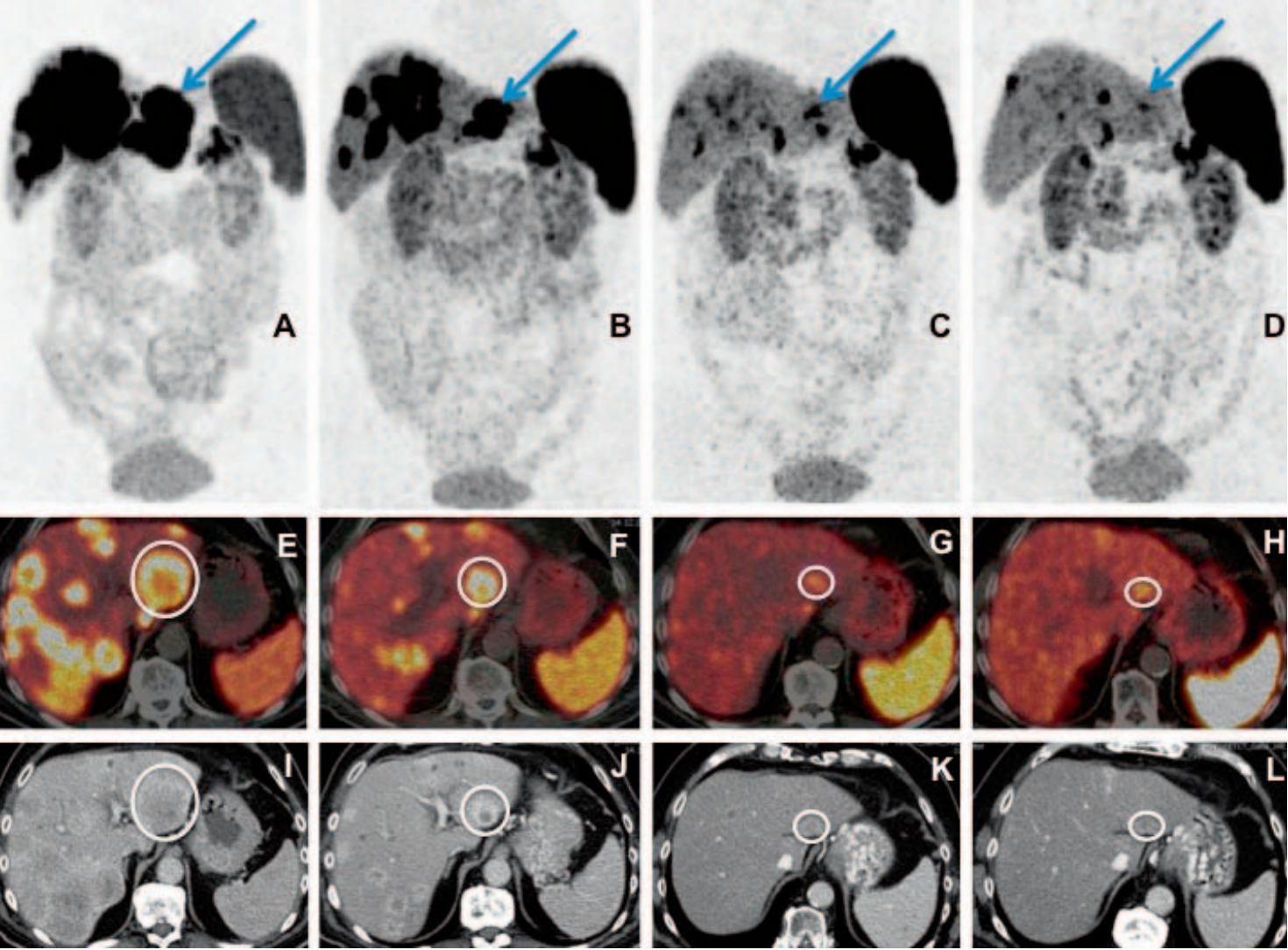
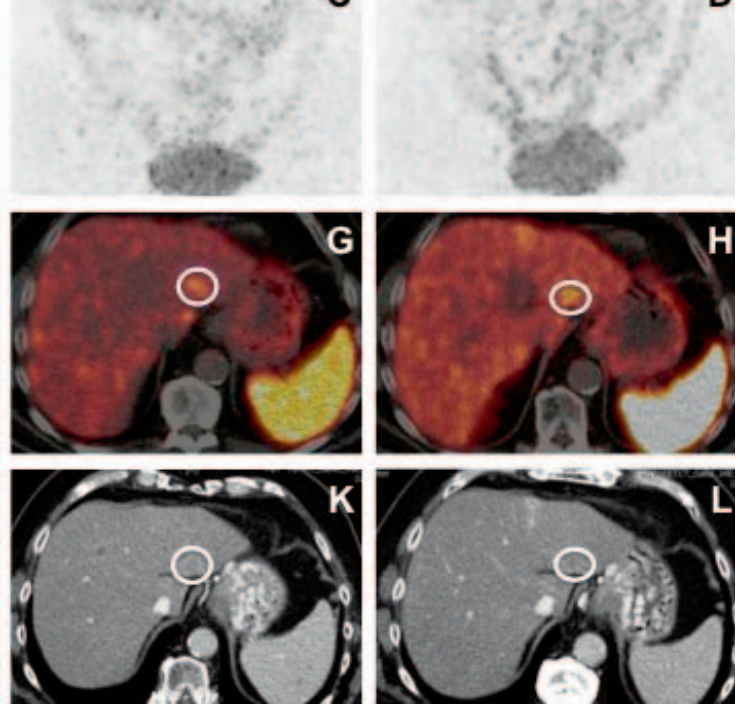

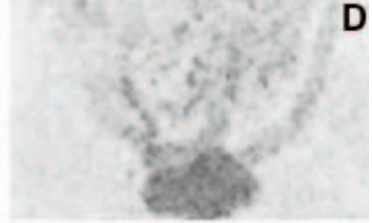

primary tumor in the

pancreas. A-D Maximum intensity projection images of PET; E-H fused transverse PET/CT images; I-L contrast-enhanced CT images in transverse view; A, E, I PET/CT before PRRT; B, F, J PET/CT after 2 cycles of PRRT; C, G, K PET/CT 4 months after the 4th cycle of PRRT; D, H, L PET/CT 1 year after the 4th cycle of PRRT. A continuing significant decrease in size and uptake of the target lesion in segment 3 of the liver (marked by arrow in the top row and by a circle in the transverse PET/CT images) is demonstrated here after 2 and 4 PRRT cycles, as well as a further reduction in uptake (delayed response) on PET/CT 1 year after $\operatorname{PRRT}(\mathbf{D}, \mathbf{H}, \mathbf{L})$.

NETs who completed three or more cycles of PRRT compared the efficacy of combined Y-90-DOTATOC and Lu-177-DOTATOC with Y-90-DOTATOC alone and found that DUO-PRRT was associated with an improved OS in patients completing three or more cycles of treatment [40].

Since the availability of Lu-177 in 2003, the Bad Berka group has pioneered the systematic use of DUO-PRRT as well as tandem PRRT in a large patient cohort of 416 patients with progressive NETs refractory to octreotide/interferon treatment or chemotherapy and showed a median OS from the time of first diagnosis of 210 months and a median survival after the first PRRT of 59 months (fig. 1). In patients with NETs of non-pancreatic origin and pancreatic NETs (pNETs) with PD, tumor response after a mean follow-up of 2 years showed that after three cycles of PRRT, complete remission, partial remission, and MR were seen in $48 \%$ of patients with non-pancreatic NETs and in $52 \%$ of patients with pNETs, whilst the disease was stabilized in $45 \%$ of patients with non-pancreatic NETs and in 39\% of patients with pNETs (fig. 2). 36 patients $(8.7 \%)$ with advanced disease died of $\mathrm{PD}$. Objective tumor responses, including improvement of clinical symptoms, were seen in $93 \%$ (91\% pNETs) of patients [41].

\section{Dosimetry}

The basis for radiation therapy is to deliver the highest possible dose to tumor tissue while sparing normal organs. Therefore, dose estimation for tumor and normal organs is essential for treatment planning and is based on the MIRD scheme [42]. Furthermore, there are interindividual differences in dose delivery, particularly given the variable metabolism or receptor density in organs and tumor lesions. This makes individual patient dosimetry absolutely necessary in PRRT. The advantage of Lu-177 is the concomitant emission of low-energy gamma rays at 208 and 113 kiloelectron volts with the therapeutic beta-radiation, which does not only allow assessment of biodistribution with post-therapy scans but also enables individualized dosimetry. It is therefore possible to calculate absorbed radiation doses to dose-limiting organs, such as kidneys and bone marrow, to better influence the decision for administering further cycles of PRRT, taking into account the cumulative administered activity. Given the highly variable SSTR densities on tumor cells and other factors like tumor size and viability, individualized dosimetry helps in patient selection and therapy planning. However, the lack of gamma emission by Y-90 results in difficulties for direct dosimetric 
Fig. 3. 65-year-old patient with metastatic non-functioning pancreatic neuroendocrine tumor first diagnosed in 2006; status after laparotomy, appendectomy, and resection of metastases, 6 courses of chemotherapy with capecitabine, right hemihepatectomy, cholecystectomy, and adhesiolysis. A total of 9 cycles of DUO peptide receptor radionuclide therapy (PRRT) (A-I whole-body posttherapy scans in anterior view after 1-9 cycles, respectively) were administered over a period of almost 9 years. The first treatment phase (September 2006 until April 2008) consisted of 5 cycles, while the second phase (6th cycle) took place in November 2009, the third phase (7th cycle) in September 2011, the fourth phase (8th cycle) in July 2013, and the fifth phase (9th cycle) in February 2015. J There was no demonstrable significant renal toxicity over 9 years; i.e., the stable tubular extraction rate was determined by Tc-

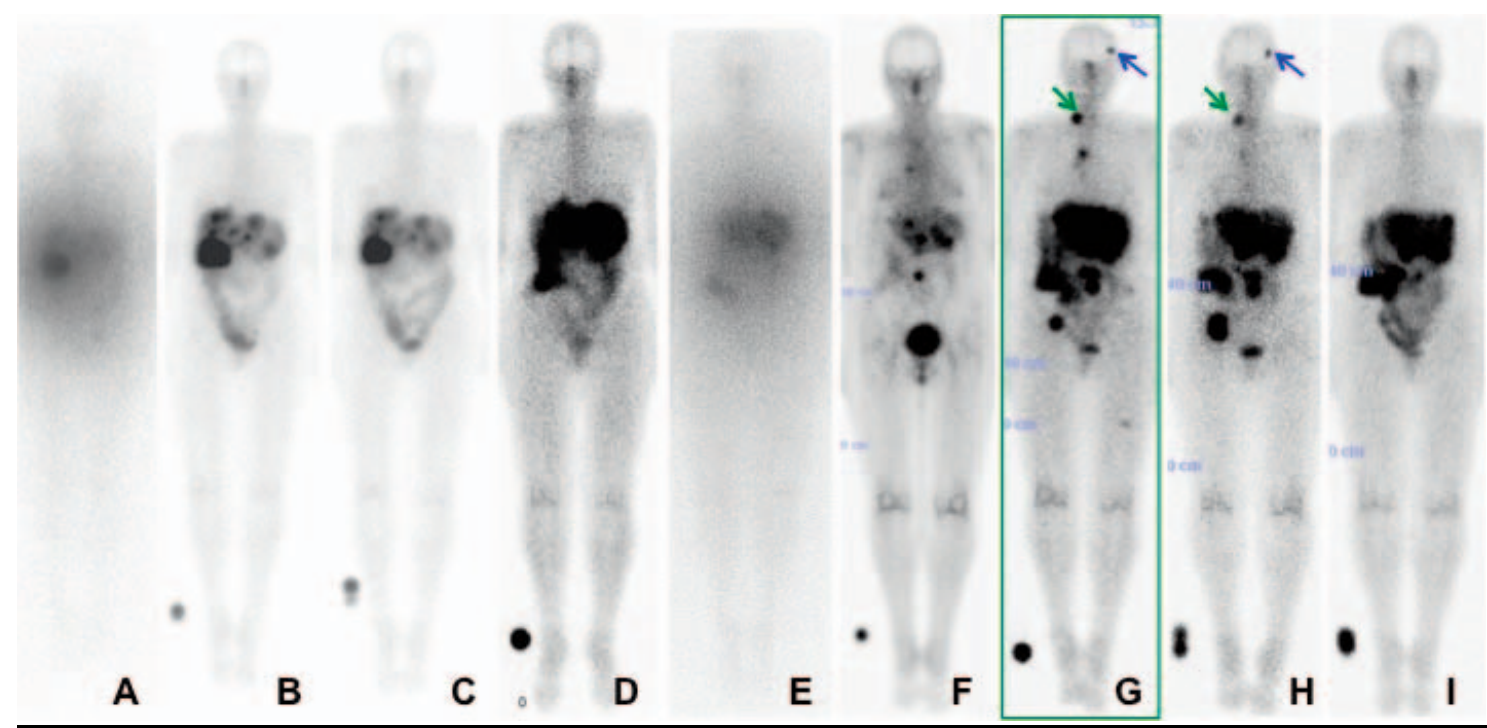
99m MAG3 scintigra-

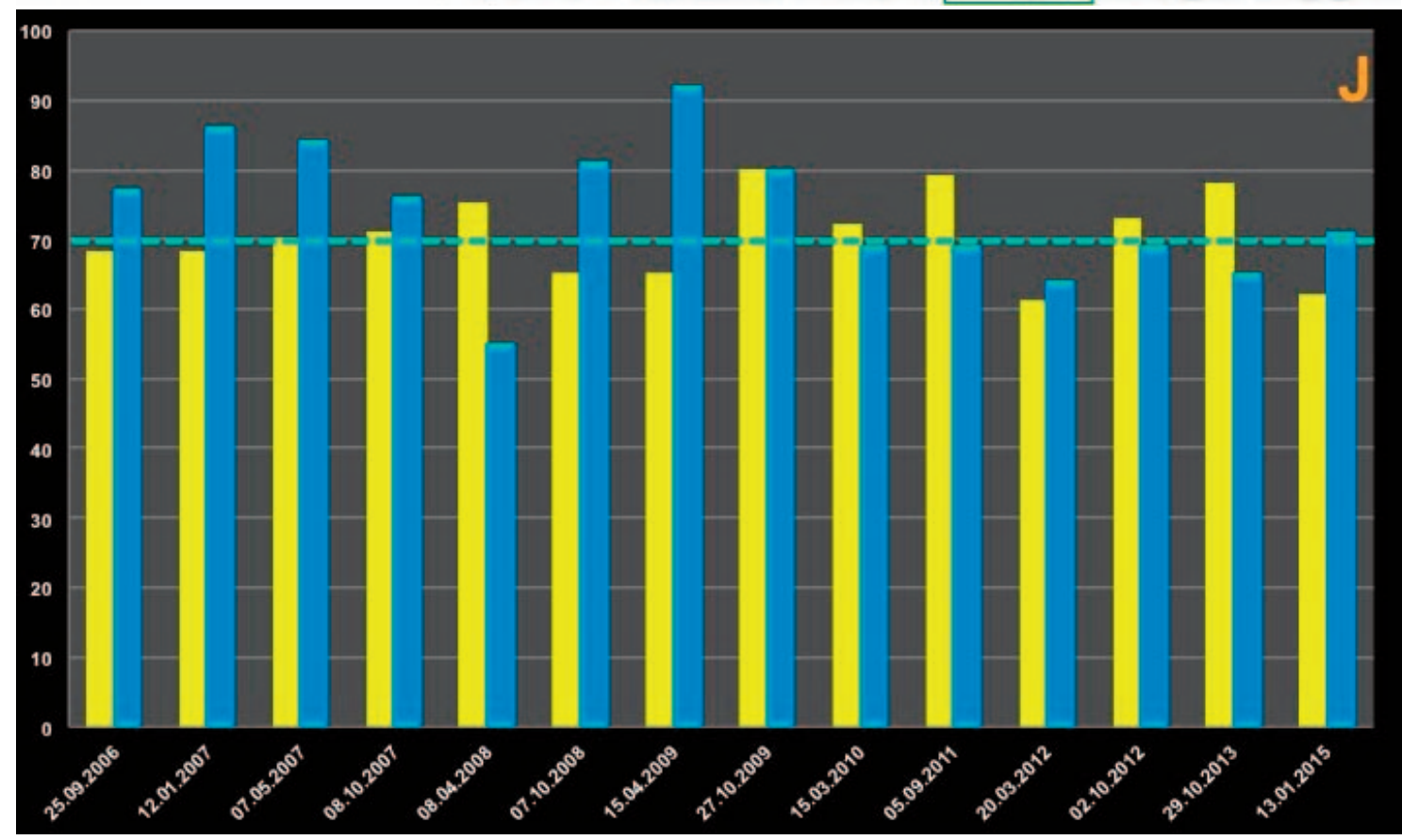

phy (yellow graphs) and glomerular filtration rate, both given in percent (the dotted line indicates the normal limit, i.e. $70 \%$ ), and was derived from using Tc-99m DTPA (blue graphs) with the single plasma sample method. In A the first and in $\mathbf{E}$ the fifth PRRT cycle Y-90 was used (the post-therapy scans appear hazy due to the poorer resolution of the Bremsstrahlung imaging), as compared to Lu-177 in the other cycles (B-D, F-I). Complete regression of the brain metastasis (blue arrow) and the right supraclavicular lymph node metastasis (green arrow) after the 7th and 8th PRRT cycle, as demonstrated in the post-therapy scan after the 9th PRRT cycle (I).

analysis post-PRRT with Y-90 agents. The data required as input for dosimetry are blood and urine samples as well as whole body scans at adequate time intervals up to at least 3 days post PRRT. Regions of interest on planar images are useful to obtain the time-activity curves. However, a much better organ-specific three-dimensional activity distribution is provided by single-photon emission computed tomography (SPECT) or SPECT/CT images, although the acquisition is time-consuming. Using dedicated software like OLINDA/EXM, mean absorbed dose estimates can be derived based on the calculated residence time of radiopeptide used [43].

\section{Treatment Side Effects}

The side effects of greatest concern regarding PRRT are potential renal and bone marrow toxicity which are described in more detail below. However, there are rarely other, relatively minor side effects which have been described, including gastrointestinal symptoms of nausea, vomiting, abdominal pain, diarrhea, as well as general fatigue, mild alopecia (rare), headache, and dizziness. 


\section{Nephrotoxicity}

The radiopeptides are filtered through glomerular capillaries in the kidneys due to their small size, and subsequently reabsorbed and retained in the proximal tubular cells, resulting in renal irradiation. Therefore, the kidneys are the potential dose-limiting organs for PRRT due to their marked radiosensitivity $[44,45]$. While the crossfire effect is beneficial for overcoming the inhomogeneity of receptor expression in cancer cells, allowing irradiation of tumor cells which are not directly targeted by the radiopharmaceutical, the long range of the Y-90 beta particles appears to further increase the potential for kidney toxicity. Cases of severe, end-stage renal disease have been reported with high activities of Y-90 at an early stage of introduction of PRRT at the beginning of this century [46]. However, adequate renal protection can minimize the risk of renal damage, and this is commonly achieved with positively charged molecules such as L-lysine and/or L-arginine which competitively inhibit the proximal tubular reabsorption of the radiopeptide and which are co-administered with PRRT. These have been shown to reduce the renal dose by $9-53 \%$ [47]. There is a further dose reduction by up to $39 \%$ when the infusion is prolonged over $10 \mathrm{~h}$ and by up to $65 \%$ when prolonging it over 2 days after radiopeptide administration, thereby providing protection almost throughout the entire elimination phase through the kidneys [44]. The patients should also be well hydrated. Gelofusine, in addition to the amino acids, also inhibits the reabsorption of these peptides and has been shown to reduce the renal uptake significantly [48]. The role of radioprotective drugs, such as amifostine, has also been investigated in preclinical studies and has been demonstrated to alleviate the radiation-induced renal damage during therapy with Lu-DOTATATE [49].

Despite renal protection, there is a very small risk of renal toxicity, usually months after irradiation, and more likely with Y-90-labeled peptides (median $7.3 \%$ per year), compared to Lu-177-labeled peptides (median 3.8\% per year) [45]. The contributing factors to the risk of renal impairment following PRRT include the cumulative and per-cycle renal absorbed dose, age, hypertension, and diabetes. The maximum tolerated renal dose in patients treated with external beam radiotherapy was considered to be 23-27 Gy [50]. For internal radiation therapy, the biologically equivalent dose (BED) was found to more accurately predict the renal toxicity, where the individual renal volume, dose rate, and fractionation, which are taken into account by the BED, were able to predict renal function impairment [51]. A much lower threshold of the BED to the kidney, i.e. $28 \mathrm{~Gy}$ in patients with risk factors (mainly hypertension and diabetes) compared to those without risk factors (40 Gy), is a predictor of renal toxicity after PRRT [52].

In a large retrospective study of 1,109 patients treated with Y90-DOTATOC found a $9 \%$ prevalence of permanent renal toxicity [11]. However, in this study, there were relatively high doses of Y-90 administered per cycle (3.7 GBq/ $\mathrm{m}^{2}$ body surface area). Fractionation of PRRT, as in the case of external beam radiotherapy, may be safer since internal radiation therapy leads to a continuous radiation delivery at a low and decreasing dose rate over time, which may allow the normal kidney tissue to repair the radiation damage [53].
In a long-term low-dose protocol, administering multiple (up to 10) cycles per patient and adequate nephroprotection (co-administering gelofusine additionally in case of Y-90), no significant renal toxicity (i.e. end-stage renal insufficiency) has been reported by the Bad Berka group in more than 1,000 patients treated with Lu-177 and/or Y-90 (>1,500 treatment cycles using Y-90) [54]. A more recent report of 1,200 patients treated with Lu-177-DOTATATE also showed no evidence of long-term renal insufficiency either (fig. 3) [35]. However, PRRT is recommended to be performed at specialized centers as NET patients require a highly individualized interdisciplinary treatment and long-term care [55].

\section{Bone Marrow Toxicity}

Although the bone marrow has been reported to be susceptible to PRRT, it does not appear to be the principal dose-limiting organ. Severe (grade 3 and 4) acute bone marrow toxicity has been reported in $10-13 \%$ of Y-90-DOTATOC treatment cycles; this is much less common with Lu-177-DOTATATE which is reported at $2-3 \%$ of cycles [21]. The risk factors for acute toxicity were patients $>70$ years of age at the beginning of treatment, prior chemotherapy, creatinine clearance (estimated with Cockcroft's formula) $\leq 60$ $\mathrm{ml} / \mathrm{min}$, and the presence of bone metastases [24].

MDS can occur in about $2 \%$ of patients, whilst acute leukemia may develop in approximately $1 \%$ of patients [52], particularly in patients receiving high bone marrow doses and if they have been previously treated with alkylating chemotherapeutic agents [56]. The risk for MDS and acute leukemia was increased in patients with previous myelotoxic chemotherapy, other bone marrow risk factors (e.g. tumor invasion of bone marrow), and grade of platelet toxicity [52]. Although the period of times between the start of PRRT and the development of MDS or acute leukemia are similar (approximately 1,500 days), these conditions were noted to develop significantly later than persistent nephrotoxicity [52].

An analysis of bone marrow dosimetry with Lu-177-DOTATATE found that the concentration of radioactivity in the bone marrow and blood was similar, with no significant binding of the radiopeptide to the stem cells, thus negating the hypothesis that high bone marrow irradiation was due to the SSTR expression on stem cells [57]. A prior study using ${ }^{111} \mathrm{In}$-octreotide and ${ }^{86} \mathrm{Y}-\mathrm{DO}-$ TATOC has shown that the red marrow uptake may be related to transchelation of the radiometal to transferrin [58].

\section{Newer Approaches to Peptide Receptor Radionuclide Therapy}

The efficacy of locoregional treatment using radiopeptides has been investigated, with the most promising approach being the intra-arterial administration of PRRT to selectively target tumor receptors in specific liver segments and to minimize systemic toxicity. An arbitrary SUV $\mathrm{max}_{\text {max }}$ cutoff of 16.4 on ${ }^{68} \mathrm{Ga}$-DOTATOC PET/ $\mathrm{CT}$ in liver metastases has been proposed to select patients for intra-arterial PRRT [59]. Intra-arterial administration of a mean cumulative activity of $58 \mathrm{GBq}$ (1,570 millicurie) ${ }^{111} \mathrm{In}$-octreotide in 16 
GEP-NET patients with liver metastases showed an objective tumor response of CR and PR in $56 \%$ of patients, with the median survival for patients with CR, PR, or SD being 32 months [60].

The radiosensitizing effect of capecitabine, an orally administered prodrug of 5-fluorouracil (5-FU), has also been studied based on evidence gained with a combination of external beam radiotherapy and chemotherapy [61-63]. The principle of using the prodrug is that many tumors require a higher amount of thymidine phosphorylase (TP) to convert the inactive form (capecitabine) into its active form (5-FU), which has led to a higher concentration of the active form in tumors than in normal tissue. Synergistically, irradiation can induce an upregulation of TP which may enhance the effect of capecitabine [64]. A randomized multicenter study compared Lu177-DOTATATE treatment with and without capecitabine in a dose of $1,650 \mathrm{mg} / \mathrm{m}^{2}$ for 14 days after each treatment cycle in patients with GEP-NETs, showing the safety and feasibility of this approach. Recently, another study using Lu-177-DOTATATE in combination with capecitabine in 33 patients with GEP-NETs showed PR in 24\% and SD in $70 \%$ of patients [61].

Neoadjuvant PRRT has also been administered to patients with inoperable NETs in order to induce radiation necrosis, thus rendering the tumor operable and subsequently resulting in complete remission after surgery [65]. Another recent study also validated the results of neoadjuvant PRRT [66]. A rationale for the adjuvant use of PRRT after surgery of NETs is to prevent metastases due to the tumor spill as a result of surgical handling.

\section{Future Perspectives}

SSTR antagonists may be superior to agonists due to higher receptor affinity and can result in better tumor targeting. They are currently being investigated in a clinical trial. Likewise, somatostatin analogs targeting all known SSTR subtypes (except SSTR4) - and therefore a wider tumor spectrum - as well as targeting of multiple receptors are under investigation. Future clinical studies will also address the efficacy and safety of combining PRRT with TACE, radiofrequency ablation, chemotherapy agents (other than capecitabine, e.g. temazolomide or doxorubicin), kinase inhibitors such as everolimus, sunitinib or sorafenib, and also antibodies such as bevacizumab [67]. There are also preliminary results using alpha-emitters such as ${ }^{213} \mathrm{Bi}$ - or ${ }^{225} \mathrm{Ac}$-labeled DOTATOC, where therapeutic effects were observed in several patients refractory to treatment with beta-emitters and represent promising new treatment options in NET patients refractory to the standard therapies [68].

\section{Conclusion}

There is increasing evidence that PRRT is the treatment of choice for advanced or progressive, SSTR-positive NETs, with benefits in PFS and OS as well as with a significant improvement in clinical symptoms. In patients with progressive NETs, fractionated, personalized PRRT with lower amounts of radioactivity given over a longer period of time results in good therapeutic responses and avoids severe hematological and/or renal toxicity, therefore improving quality of life.

\section{Disclosure Statement}

The authors declare that they have no conflict of interest.

\section{References}

1 Bosman FT, Carneiro F, Hruban RH, Theise ND: WHO Classification of Tumors of the Digestive System. Lyon, IARC Press, 2010.

$\checkmark 2$ Phan AT, Öberg K, Choi J, et al.; North American Neuroendocrine Tumor Society (NANETS): NANETS consensus guideline for the diagnosis and management of neuroendocrine tumors: well-differentiated neuroendocrine tumors of the thorax (includes lung and thymus). Pancreas 2010;39:784-798

3 Severi S, Grassi I, Nicolini S, et al: Peptide receptor radionuclide therapy in the management of gastrointestinal neuroendocrine tumors: efficacy profile, safety, and quality of life. Onco Targets Ther 2017;10:551557.

4 Kaemmerer D, Peter L, Lupp A, et al: Molecular imaging with ${ }^{68} \mathrm{Ga}$-SSTR PET/CT and correlation to immunohistochemistry of somatostatin receptors in neuroendocrine tumors. Eur J Nucl Med Mol Imaging 2011;38:1659-1668.

5 Modlin IM, Pavel M, Kidd M, Gustafsson BI: Review article: somatostatin analogs in the treatment of gastroenteropancreatic neuroendocrine (carcinoid) tumors. Aliment Pharmacol Ther 2010;31:169-188.
6 Hofman MS, Kong G, Neels OC, et al: High management impact of Ga-68 DOTATATE (GaTate) PET/CT for imaging neuroendocrine and other somatostatin expressing tumors. J Med Imaging Radiat Oncol 2012; 56:40-47.

7 Prasad V, Steffen IG, Pavel M, et al: Somatostatin receptor PET/CT in restaging of typical and atypical lung carcinoids. EJNMMI Res 2015;5:53.

8 Kulkarni HR, Baum RP: Patient selection for personalized peptide receptor radionuclide therapy using Ga-68 somatostatin receptor PET/CT. PET Clin 2014; 9:83-90.

9 Kulkarni HR, Baum RP: Theranostics with Ga-68 somatostatin receptor PET/CT: monitoring response to peptide receptor radionuclide therapy. PET Clin 2014; 9:91-97.

10 Valkema R, De Jong M, Bakker WH, et al: Phase I study of peptide receptor radionuclide therapy with [ ${ }^{111}$ In-DTPA ${ }^{0}$ octreotide: the Rotterdam experience. Semin Nucl Med 2002;32:110-122.

11 Imhof A, Brunner P, Marincek N, et al: Response, survival, and long-term toxicity after therapy with the radiolabeled somatostatin analog $\left[{ }^{90} \mathrm{Y}\right.$-DOTA $]$-TOC in metastasized neuroendocrine cancers. J Clin Oncol 2011;29:2416-2423.
12 Reubi JC, Schär JC, Waser B, et al: Affinity profiles for human somatostatin receptor subtypes SST1-SST5 of somatostatin radiotracers selected for scintigraphic and radiotherapeutic use. Eur J Nucl Med 2000;27: 273-282.

13 Wild D, Schmitt JS, Ginj M, et al: DOTA-NOC, a highaffinity ligand of somatostatin receptor subtypes 2, 3 and 5 for labelling with various radiometals. Eur J Nucl Med Mol Imaging 2003;30:1338-1347.

14 Kulkarni HR, Schuchardt C, Baum RP: Peptide receptor radionuclide therapy with Lu-177 labeled somatostatin analogs DOTATATE and DOTATOC: contrasting renal dosimetry in the same patient. Recent Results Cancer Res 2013;194:551-559.

15 Wehrmann C, Senftleben S, Zachert C, Muller D, Baum RP: Results of individual patient dosimetry in peptide receptor radionuclide therapy with ${ }^{177} \mathrm{Lu}$ DOTA-TATE and ${ }^{177} \mathrm{Lu}$ DOTA-NOC. Cancer Biother Radiopharm 2007;22:406-416.

16 Cremonesi M, Ferrari M, Di Dia A, et al: Recent issues on dosimetry and radiobiology for peptide receptor radionuclide therapy. Q J Nucl Med Mol Imaging 2011;55:155-167. 
17 Bodei L, Kidd M, Modlin IM, et al: Measurement of circulating transcripts and gene cluster analysis predicts and defines therapeutic efficacy of peptide receptor radionuclide therapy (PRRT) in neuroendocrine tumors. Eur J Nucl Med Mol Imaging 2016;43:839-851.

18 Severi S, Nanni O, Bodei L, et al: Role of ${ }^{18}$ FDG PET/ $\mathrm{CT}$ in patients treated with ${ }^{177} \mathrm{Lu}$-DOTATATE for advanced differentiated neuroendocrine tumors. Eur J Nucl Med Mol Imaging 2013;40:881-888.

19 Campana D, Capurso G, Partelli S, et al: Radiolabeled somatostatin analog treatment in gastroenteropancreatic neuroendocrine tumors: factors associated with response and suggestions for therapeutic sequence. Eur J Nucl Med Mol Imaging 2013;40:1197-1205.

20 Kesavan M, Claringbold PG, Turner JH: Hematological toxicity of combined ${ }^{177} \mathrm{Lu}$-octreotate radiopeptide chemotherapy of gastroenteropancreatic neuroendocrine tumors in long-term follow-up. Neuroendocrinology 2014;99:108-117.

21 Bodei L, Mueller-Brand J, Baum RP, et al: The joint IAEA, EANM, and SNMMI practical guidance on peptide receptor radionuclide therapy (PRRNT) in neuroendocrine tumors. Eur J Nucl Med Mol Imaging 2013;40:800-816.

22 Bodei L, Ferone D, Grana CM, et al: Peptide receptor therapies in neuroendocrine tumors. J Endocrinol Invest 2009;32:360-369.

23 Bushnell DL Jr, O’Dorisio TM, O’Dorisio MS, et al: ${ }^{90} \mathrm{Y}$-edotreotide for metastatic carcinoid refractory to octreotide. J Clin Oncol 2010;28:1652-1659.

24 Kwekkeboom DJ, de Herder WW, Kam BL, et al: Treatment with the radiolabeled somatostatin analog $\left[{ }^{177} \mathrm{Lu}-\mathrm{DOTA}{ }^{0}, \mathrm{Tyr}^{3}\right]$ octreotate: toxicity, efficacy, and survival. J Clin Oncol 2008;26:2124-2130.

25 Kwekkeboom DJ, Kam BL, van Essen M, et al: Somatostatin-receptor-based imaging and therapy of gastroenteropancreatic neuroendocrine tumors. Endocr Relat Cancer 2010;17:R53-73

26 Bartolomei M, Bodei L, De Cicco C, et al: Peptide receptor radionuclide therapy with Y-90-DOTATOC in recurrent meningioma. Eur J Nucl Med Mol Imaging 2009;36:1407-1416.

27 Bodei L, Handkiewicz-Junak D, Grana C, et al: Receptor radionuclide therapy with Y-90-DOTATOC in patients with medullary thyroid carcinomas. Cancer Biother Radiopharm 2004;19:65-71.

28 Chang CA, et al: ${ }^{68} \mathrm{Ga}$-DOTATATE and ${ }^{18} \mathrm{~F}$-FDG PET/ CT in paraganglioma and pheochromocytoma: utility, patterns and heterogeneity. Cancer Imaging 2016;16:22.

29 Iten F, et al: Response to [ ${ }^{90}$ Yttrium-DOTA]-TOC treatment is associated with long-term survival benefit in metastasized medullary thyroid cancer: a phase II clinical trial. Clin Cancer Res 2007;13:6696-6702.

30 Krenning EP, Kooij PP, Bakker WH, et al: Radiotherapy with a radiolabeled somatostatin analog, $\left[{ }^{111} \mathrm{In}\right.$ DTPA-D-Phe ${ }^{1}$-octreotide. A case history. Ann N Y Acad Sci 1994;733:496-506.

31 Hörsch D, Ezziddin S, Haug A, et al: Effectiveness and side-effects of peptide receptor radionuclide therapy for neuroendocrine neoplasms in Germany: a multiinstitutional registry study with prospective follow-up. Eur J Cancer 2016;58:41-51.

- 32 Ezziddin S, Attassi M, Yong-Hing CJ, et al: Predictors of long-term outcome in patients with well-differentiated gastroenteropancreatic neuroendocrine tumors after peptide receptor radionuclide therapy with ${ }^{177} \mathrm{Lu}$ octreotate. J Nucl Med 2014;55:183-190.

33 Paganelli G, Sansovini M, Ambrosetti A, et al: ${ }^{177} \mathrm{Lu}-$ Dota-octreotate radionuclide therapy of advanced gastrointestinal neuroendocrine tumors: results from a phase II study. Eur J Nucl Med Mol Imaging 2014;41: 1845-1851.
34 Baum RP, Kluge AW, Kulkarni $\mathrm{H}$, et al: $\left[{ }^{177} \mathrm{Lu}-\right.$ DOTA $]^{0}$-D-Phe ${ }^{1}-$ Tyr $^{3}$-Octreotide $\left({ }^{177}\right.$ Lu-DOTATOC) for peptide receptor radiotherapy in patients with advanced neuroendocrine tumors: a phase-II study. Theranostics 2016;6:501-510.

35 Brabander T, van der Zwaan WA, Teunissen JJM, et al: Long-term efficacy, survival, and safety of $\left[{ }^{177} \mathrm{Lu}-\right.$ DOTA $^{0}, \mathrm{Tyr}^{3}$ ]octreotate in patients with gastroenteropancreatic and bronchial neuroendocrine tumors. Clin Cancer Res 2017;23:4617-4624.

36 Strosberg J, El-Haddad G, Wolin E, et al: Phase 3 trial of ${ }^{177} \mathrm{Lu}$-Dotatate for midgut neuroendocrine tumors. N Engl J Med 2017;376:125-135.

37 O’Donoghue JA, Bardies M, Wheldon TE: Relationships between tumor size and curability for uniformly targeted therapy with beta-emitting radionuclides. J Nucl Med 1995;36:1902-1909.

38 de Jong, M, Breeman WA, Valkema R, Bernard BF, Krenning EP: Combination radionuclide therapy using ${ }^{177} \mathrm{Lu}$ - and ${ }^{90} \mathrm{Y}$-labeled somatostatin analogs. J Nucl Med 2005;46(suppl 1):13S-17S.

39 Kunikowska J, Królicki L, Hubalewska-Dydejczyk A, et al: Clinical results of radionuclide therapy of neuroendocrine tumors with ${ }^{90} \mathrm{Y}$-DOTATATE and tandem ${ }^{90} \mathrm{Y} /{ }^{177} \mathrm{Lu}$-DOTATATE: which is a better therapy option? Eur J Nucl Med Mol Imaging 2011;38:1788-1797.

40 Villard L, Romer A, Marincek N, et al: Cohort study of somatostatin-based radiopeptide therapy with $\left[{ }^{90} \mathrm{Y}\right.$ DOTA $]$-TOC versus $\left[{ }^{90} \mathrm{Y}-\mathrm{DOTA}\right]-$ TOC plus $\left[{ }^{177} \mathrm{Lu}-\right.$ DOTA]-TOC in neuroendocrine cancers. J Clin Oncol 2012;30:1100-1106.

41 Baum RP, Kulkarni HR, Carreras C: Peptides and receptors in image-guided therapy: theranostics for neuroendocrine neoplasms. Semin Nucl Med 2012;42:190-207.

42 Siegel JA, Thomas SR, Stubbs JB, et al: MIRD pamphlet no. 16: techniques for quantitative radiopharmaceutical biodistribution data acquisition and analysis for use in human radiation dose estimates. J Nucl Med 1999; 40:37S-61S.

43 Stabin MG, Sparks RB, Crowe E: OLINDA/EXM: the second-generation personal computer software for internal dose assessment in nuclear medicine. J Nucl Med 2005;46:1023-1027.

44 Bodei L, Cremonesi M, Grana C, et al: Receptor radionuclide therapy with ${ }^{90} \mathrm{Y}$-[DOTA $]^{0}-\mathrm{Tyr}^{3}$-octreotide $\left({ }^{90} \mathrm{Y}\right.$-DOTATOC) in neuroendocrine tumors. Eur J Nucl Med Mol Imaging 2004;31:1038-1046.

45 Valkema R, Pauwels SA, Kvols LK, et al: Long-term follow-up of renal function after peptide receptor radiation therapy with ${ }^{90} \mathrm{Y}$-DOTA ${ }^{0}, \mathrm{Tyr}^{3}$-octreotide and ${ }^{177}$ Lu-DOTA $^{0}$, Tyr $^{3}$-octreotate. J Nucl Med 2005; 46(suppl 1):83S-91S.

46 Cybulla M, Weiner SM, Otte A: End-stage renal disease after treatment with ${ }^{90} \mathrm{Y}$-DOTATOC. Eur J Nucl Med 2001;28:1552-1554

47 Rolleman EJ, Valkema R, de Jong M, Kooij PP, Krenning EP: Safe and effective inhibition of renal uptake of radiolabeled octreotide by a combination of lysine and arginine. Eur J Nucl Med Mol Imaging 2003;30:9-15.

48 Rolleman EJ, Bernard BF, Breeman WA, et al: Molecular imaging of reduced renal uptake of radiolabeled [DOTA ${ }^{0}$, $\mathrm{Tyr}^{3}$ ]octreotate by the combination of lysine and Gelofusine in rats. Nuklearmedizin 2008;47:110-115.

49 Rolleman EJ, Forrer F, Bernard B, et al: Amifostine protects rat kidneys during peptide receptor radionuclide therapy with $\left[{ }^{177} \mathrm{Lu}-\mathrm{DOTA}{ }^{0}, \mathrm{Tyr}^{3}\right]$ octreotate. Eur J Nucl Med Mol Imaging 2007;34:763-771.

50 Cassady JR: Clinical radiation nephropathy. Int J Radiat Oncol Biol Phys 1995;31:1249-1256.

51 Barone R, Borson-Chazot F, Valkema R, et al: Patientspecific dosimetry in predicting renal toxicity with ${ }^{90} \mathrm{Y}-$ DOTATOC: relevance of kidney volume and dose rate in finding a dose-effect relationship. J Nucl Med 2005; 46(suppl 1):99S-106S.
52 Bodei L, Cremonesi M, Ferrari M, et al: Long-term evaluation of renal toxicity after peptide receptor radionuclide therapy with ${ }^{90} \mathrm{Y}$-DOTATOC and ${ }^{177} \mathrm{Lu}$-DOTATATE: the role of associated risk factors. Eur J Nucl Med Mol Imaging 2008;35:1847-1856.

53 Dale R, Carabe-Fernandez A: The radiobiology of conventional radiotherapy and its application to radionuclide therapy. Cancer Biother Radiopharm 2005;20:47-51.

54 Baum RP, Kulkarni HR: THERANOSTICS: from molecular imaging using Ga-68 labeled tracers and PET/ CT to personalized radionuclide therapy - the Bad Berka experience. Theranostics 2012;2:437-447.

55 Hörsch D, Kulkarni HR, Baum RP: THERANOSTICS - clinical aimshots in surgical warfare against well-differentiated neuroendocrine neoplasms. Ann Transl Med 2014;2:1.

56 Kwekkeboom DJ, Mueller-Brand J, Paganelli G, et al: Overview of results of peptide receptor radionuclide therapy with 3 radiolabeled somatostatin analogs. J Nucl Med 2005;46(suppl 1):62S-66S.

57 Forrer F, Krenning EP, Kooij PP, et al: Bone marrow dosimetry in peptide receptor radionuclide therapy with $\left[{ }^{177} \mathrm{Lu}-\mathrm{DOTA}{ }^{0}, \mathrm{Tyr}^{3}\right]$ octreotate. Eur J Nucl Med Mol Imaging 2009;36:1138-1146.

58 Walrand S, Barone R, Pauwels S, Jamar F: Experimental facts supporting a red marrow uptake due to radiometal transchelation in ${ }^{90} \mathrm{Y}$-DOTATOC therapy and relationship to the decrease of platelet counts. Eur J Nucl Med Mol Imaging 2011;38:1270-1280.

59 Kratochwil C, Stefanova M, Mavriopoulou E, et al: SUV of $\left[{ }^{68} \mathrm{Ga}\right]$ DOTATOC-PET/CT predicts response probability of PRRT in neuroendocrine tumors. Mol Imaging Biol 2015;17:313-318.

60 Limouris GS, Chatziioannou A, Kontogeorgakos D, et al: Selective hepatic arterial infusion of In-111-DTPA$\mathrm{Phe}^{1}$-octreotide in neuroendocrine liver metastases. Eur J Nucl Med Mol Imaging 2008;35:1827-1837.

61 Claringbold PG, Brayshaw PA, Price RA, Turner JH: Phase II study of radiopeptide ${ }^{177} \mathrm{Lu}$-octreotate and capecitabine therapy of progressive disseminated neuroendocrine tumors. Eur J Nucl Med Mol Imaging 2011;38:302-311.

62 Rich TA, Shepard RC, Mosley ST: Four decades of continuing innovation with fluorouracil: current and future approaches to fluorouracil chemoradiation therapy. J Clin Oncol 2004;22:2214-2232.

63 van Essen M, Krenning EP, Kam BL, et al: Report on short-term side effects of treatments with ${ }^{177} \mathrm{Lu}$-octreotate in combination with capecitabine in seven patients with gastroenteropancreatic neuroendocrine tumors. Eur J Nucl Med Mol Imaging 2008;35:743-748.

64 Sawada N, Ishikawa T, Sekiguchi F, Tanaka Y, Ishitsuka $\mathrm{H}$ : X-ray irradiation induces thymidine phosphorylase and enhances the efficacy of capecitabine (Xeloda) in human cancer xenografts. Clin Cancer Res 1999;5:2948-2953.

65 Kaemmerer D, Prasad V, Daffner W, et al: Neoadjuvant peptide receptor radionuclide therapy for an inoperable neuroendocrine pancreatic tumor. World J Gastroenterol 2009;15:5867-5870.

66 Sowa-Staszczak A, Pach D, Chrzan R, et al: Peptide receptor radionuclide therapy as a potential tool for neoadjuvant therapy in patients with inoperable neuroendocrine tumors (NETs). Eur J Nucl Med Mol Imaging 2011;38:1669-1674.

67 Horsch D, Bert T, Schrader J, et al: Pancreatic neuroendocrine neoplasms. Minerva Gastroenterol Dietol 2012;58:401-426.

68 Kulkarni HR, Singh A, Baum RP: Advances in the diagnosis of neuroendocrine neoplasms. Semin Nucl Med 2016;46:395-404. 